\title{
SIMULTANEOUS OPTIMIZATION OF STRUCTURAL SHAPE AND CONTROL SYSTEM OF LARGE-SCALE SPACE FRAME BASED ON SINE WAVE INPUTS
}

\author{
M. DAN \& M. KOHIYAMA \\ Graduate School of Science and Technology, Keio University, Japan.
}

\begin{abstract}
This paper proposes a simultaneous optimal design method of asymmetric large-scale space frames with tuned mass dampers (TMDs). The objective function is defined by the maximum absolute acceleration response of the structure to input ground motions of sine waves. Sine waves of periods with the five natural periods having large modal participation factors of the structure are input, and the maximum responses are calculated by time-history response analysis to evaluate the objective function. The shape of the space frame, i.e. nodal coordinates of the space frame's joints, is described by a Bézier surface to reduce the number of design variables. The change from the initial values of the nodal coordinates is constrained to preserve the initial design shape, which is provided by an architect. The method employs a genetic algorithm in optimization. In addition, a case study is conducted for an asymmetric steel space frame of a vault-like shape. The results confirm the reduction of maximum absolute acceleration responses in the optimal shapes not only to the five sine waves but also to four scaled ground motion records. Moreover, the presence of TMDs enables the reduction of the peak response value and maintains similarity to the initial shape.
\end{abstract}

Keywords: architectural design, Bézier surface, earthquake engineering, genetic algorithm, optimization, seismic control, structural engineering, structural shape, space frame, tuned mass damper.

\section{INTRODUCTION}

In the design of large-scale space frames, the acceleration response and structural deformation should be reduced during an earthquake to minimize damages and improve the usability after such an event. However, accidental collapses and other damage to ceilings, as well as lighting equipment suspended from roof structures, occur often during earthquakes, such as the 1995 Kobe earthquake [1] and the 2011 Great East Japan earthquake [2]. Large-scale space frame structures, such as gymnasiums and stadiums, often serve as evacuation centers during such disasters. In addition, higher seismic performance is expected from such emergency disaster facilities. These types of structures sometimes have esthetic shapes, developed by architectural designers. Hence, sufficient seismic performance against severe ground motion is desired while maintaining unique and impressive building designs.

Vibration control devices are effective solutions for achieving these demands. A tuned mass damper (TMD) is one of the most popular seismic control systems. There have been many studies about the design and use of TMD in various structures. For example, Kaynia et al. [3] analyzed the effectiveness of a TMD against seismic inputs. Lin et al. [4] devised an optimal design method for a TMD by minimizing the mean-square displacement response ratio between structures with and without the installation of TMDs under earthquake excitation. Johnson et al. [5] proposed to use a rooftop substructure as a TMD and showed that it can effectively reduce the acceleration response of the main structure. Kusunoki et al. [6] devised a methodology to realize the seismic control of a symmetric dorm structure using a TMD. Yoshinaka and Kawaguchi [7] proposed a seismic control system using multiple TMDs. However, these studies focused on symmetric structural models, such as dorm struc- 
tures so that the application of the TMD to large-scale structures with various shapes has not been confirmed.

In addition, several studies have been conducted to design a dome shell and a space frame using optimization schemes. For example, Ramm et al. [8] proposed methods of structural optimization for the form finding and thickness design, and demonstrated the versatility of optimization schemes in shell design, such as the tuning of a bell and the form finding of a classical reinforced concrete dome shell. Ohsaki et al. [9] proposed a design method for a double-layer space truss, which is described by a parametric surface including a Bézier surface.

With respect to the optimization method, Goldberg [10] stated that genetic algorithms (GAs) have four differences compared with other classical optimization methods: (1) GAs manipulate coded versions of the problem parameters instead of the parameters themselves; thus, GAs find approximate solutions, (2) while almost all conventional methods search from a single point, GAs always operate on a whole population of points, and it improves the robustness of the algorithm, while reducing the risk of becoming trapped in a local stationary point, (3) normal GAs do not use any auxiliary information about the objective function value such as derivatives; therefore, they can be applied to any kind of continuous or discrete optimization problem, and (4) GAs use probabilistic transition operators, while conventional methods for continuous optimization apply deterministic transition operators; more specifically, there is some randomness to the way in which a new generation is computed from the actual one. Because of these advantages, GAs are preferred in a wide range of optimization problems and have been used in many structural optimization studies [11-16].

The authors proposed a design method for a large-scale space frame that simultaneously optimizes the structural shape and control system of the structure using a GA in the conference paper [17]. As an objective function of the optimization problem, the proposed method used the maximum acceleration response under some specific ground motions, and we adopted two sine waves with different periods, which are the natural periods with the first and second largest modal participation factors. In this paper, the proposed method is modified and the five natural periods with large modal participation factors are adopted for periods with input sine waves. Then, the effectiveness of the modified method is analyzed to confirm that it provides satisfactory performance in terms of the reduction of the seismic response.

\section{STRUCTURAL MODELING}

\subsection{Bézier surface}

In this paper, a space frame is modeled using finite elements. In general, a space frame has a large number of joints (nodes), and it is not efficient when performing optimization directly using all node coordinates as design variables. Hence, a two-dimensional Bézier surface is utilized to reduce the number of design variables involved in the optimization. The control points of a Bézier surface are used as design variables, and the coordinate of each node is calculated based on the equation of the Bézier surface.

The initial shape is designed in the design space, which has 50-m length in $\mathrm{X}$ direction, $30-\mathrm{m}$ width in $\mathrm{Y}$ direction, and $15 \mathrm{~m}$ in $\mathrm{Z}$ direction. The initial shape of the structure model is shown in Fig. 1. Asymmetric roof structures are adopted as the initial design, and the related shape constraints are introduced in the optimization. 


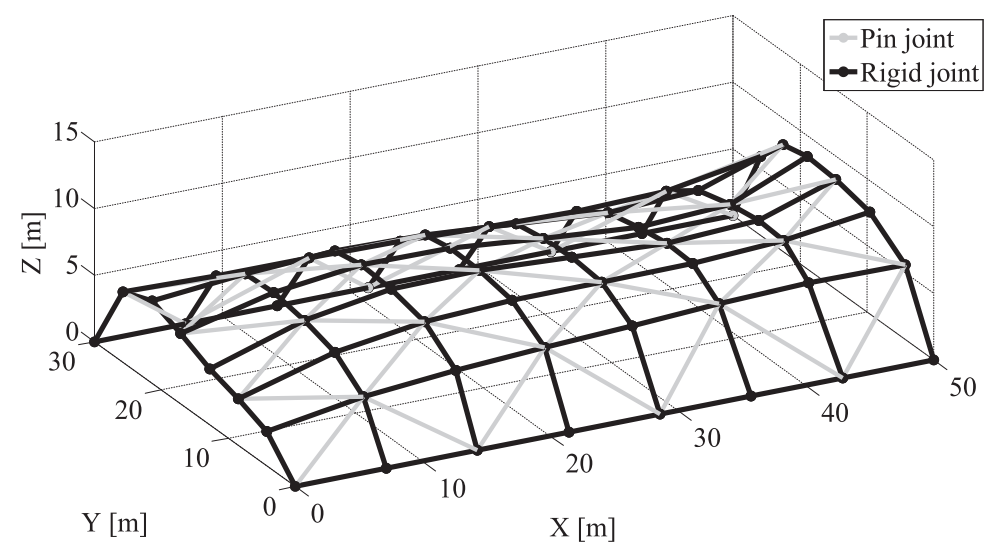

Figure 1: Initial shape.

A given Bézier surface of order $(n, m)$ is defined by a set of $(n+1)(m+1)$ control points $\mathbf{k}_{i, j}$. A two-dimensional Bézier surface can be defined as a parametric surface, on which the position vector of a point $\mathbf{p}$ is given as a function of the parametric coordinates $u$ and $v$, as follows:

$$
\mathbf{p}(u, v)=\sum_{i=0}^{n} \sum_{j=0}^{m} B_{i}^{n}(u) B_{j}^{m}(v) \mathbf{k}_{i, j},
$$

where

$$
B_{i}^{n}(u)=\left(\begin{array}{c}
n \\
i
\end{array}\right) u^{i}(1-u)^{n-i}
$$

and

$$
\left(\begin{array}{l}
n \\
i
\end{array}\right)=\frac{n !}{i !(n-i) !}
$$

are a Bernstein polynomial and binomial coefficient, respectively.

In this paper, both $n$ and $m$ are set to 4 so that the described Bézier surface has the order (4, 4). As seen in the initial shapes of the structural models shown in Fig. 1, the model has eight continuous beams in both X- and Y-directions, and there are 64 nodes in total. Using a Bézier surface of order $(4,4)$, the total number of control points is 25 . Thus, the number of design variables is substantially reduced to less than half.

\subsection{Structural parameters}

Steel tubes are assumed to be the structural members, and the continuous beams are rigidly connected. In addition to the continuous beams, diagonal beams are inserted with pin joints, as shown in Fig. 1. The total number of members is 161, and each member is modeled with a single element of a linear elastic Euler beam with distributed mass. The external diameter, thickness, and mass density of each beam are $300 \mathrm{~mm}, 15 \mathrm{~mm}$, and $7.85 \times 10^{3} \mathrm{~kg} / \mathrm{m}^{3}$, respectively. The Young modulus and Poisson ratio are set to $206 \mathrm{GPa}$ and 0.3 , respectively. 


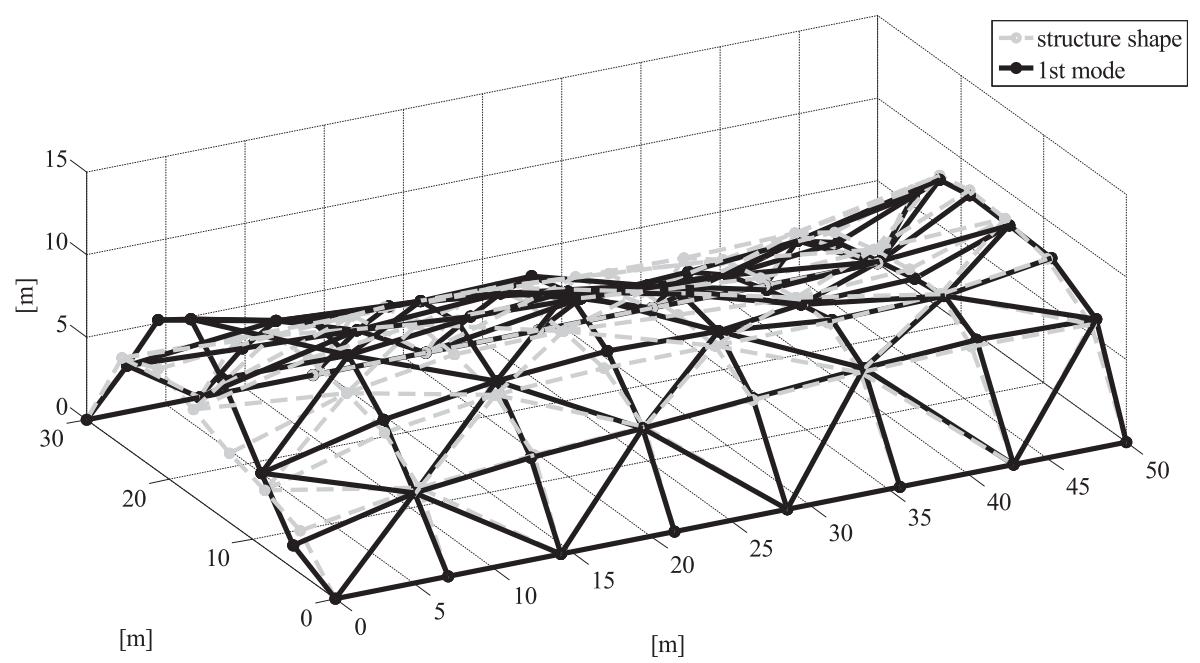

Figure 2: Fundamental mode shape of the initial shape.

Table 1: Natural periods of lower modes of initial shape.

\begin{tabular}{lccccc}
\hline Mode number & 1st & 2nd & 3rd & 4 th & 5th \\
Natural period (s) & 0.3948 & 0.2439 & 0.2286 & 0.1821 & 0.1361 \\
\hline
\end{tabular}

The Rayleigh damping is used for structural damping, and the damping factors of both the first and second modes (translational modes in the Y-direction) are set to 0.02. In this study, only roof structures are considered, and all endpoint joint nodes in the Y-direction are assumed to be supported by the rigid substructure with pin joints. Regarding the dead load, only the self-weight is considered for simplicity.

Figure 2 displays the fundamental mode shape of the initial shape. The modal shape of an asymmetric structure is complex, as all modes from the first order to the highest order consist of mixed modes of three-dimensional translation and torsion. Thus, the classification of vibration mode types is difficult.

The natural periods (first to fifth modes) of initial shape are shown in Table 1.

\section{CONTROLLER MODELING}

\subsection{Control device}

Numerous seismic control systems have been designed for buildings, and they can be classified into active, semi-active, and passive control systems depending on the presence of a power input. In general, semi-active and active control systems are more costly than passive ones because they need electrical power, sensors, and computers, which have to be repaired or replaced within a specific number of years. This paper focuses on passive control systems from a financial point of view. In previous studies, such as in [6] and [7], the effectiveness of a TMD on seismic control has been confirmed for a symmetric space frame. Thus, the same type of TMD is considered in this paper. 


\subsection{Design of tuned mass damper}

In general, the design of a TMD is determined by first deciding the mass ratio $\mu$ of the counter weight of the TMD to the whole structure including the TMD and objective controlled modes. To derive the optimal parameters of the TMD, such as its mass, damping, and stiffness, a method using the fixed point theory proposed by Den Hartog [18], together with other related methods based on the fixed point theory (e.g. Ikago et al. [19]), are well known. However, direct application of these methods to an asymmetric structure using multiple TMDs is not easy.

Thus, this paper proposes a design optimization method for a passive control system with multiple TMDs using GAs. The mass, damping, and stiffness of TMDs are included in the design variables, in addition to the control points of a Bézier surface, and their values are directly derived during the optimization.

As an example, this paper considers a seismic control system using two TMDs. The objective controlled mode of each TMD is selected based on the largest modal participation factor. Generally, the vertical seismic excitation of space frames is larger than that of normal buildings (e.g. Kato et al. [20]). Thus, it is important to consider vertical excitation in the design of a space frame. In this paper, the TMDs are set up on joint nodes that have the maximum amplitude in the objective mode vector, and the TMDs are assumed to only move in the vertical direction.

\section{OPTIMIZATION PROBLEM SETTINGS}

\subsection{Objective function}

In this paper, an optimization problem is addressed to minimize the maximum absolute acceleration response to sine wave inputs of the roof structure of a large-scale space frame. It has been reported that the design a of the TMD system based on the response to sine wave inputs is preferable because the analysis result does not depend on the properties of the external force [6]. Thus, the responses to sine wave inputs are adopted as an objective function and the optimization aims to minimize the response value. In this study, sine wave ground acceleration with the same period, phase, and amplitude is applied in the X-, Y-, and Z-directions simultaneously.

The method proposed in the previous paper uses two sine waves with different periods as input waves. The periods of input sine waves are selected according to the natural periods and modal participation factors of the structure. In the calculation, inputs are only applied in the translational direction and not in the rotational direction. Thus, the value of the distribution vector of the ground acceleration is 1 in translational directions and 0 in rotational directions. The modal participation factors are normalized according to the following equation:

$$
{ }_{s} \boldsymbol{u}^{T} \boldsymbol{M}_{s} \boldsymbol{u}=\sum_{i=1}^{N} m_{i} \cdot{ }_{s} u_{i}^{2}=1,
$$

where $\boldsymbol{M}$ and ${ }_{s} \boldsymbol{u}$ are the mass matrix and th eigenvector, respectively, and $N$ denotes the degrees of freedom. For example, when the mode number that has the largest modal participation factor is first, the natural period of the first mode is chosen as the period of a sine wave input. In this paper, we use five sine wave inputs, and the periods are selected from natural periods of the modes with the first to fifth largest modal participation factors. Although the 
Table 2: Periods of sine wave inputs in compared optimizations.

\begin{tabular}{llllllll}
\hline Period (s) & 0.1 & 0.2 & 0.3 & 0.5 & 1.0 & 2.0 & 5.0 \\
Duration (s) & 1.0 & 2.0 & 3.0 & 5.0 & 10 & 20 & 50 \\
\hline
\end{tabular}

number of sine wave inputs increases from two to five compared with the previous method, the calculation time changes slightly, and the seismic performance of the optimal design is significantly improved, as described later. To confirm the validity of the proposed method, in the calculation of the objective function, we compared seven optimization results that were derived using only a single sine wave input with a specific period. The seven specific periods of the sine wave are shown in Table 2. According to Table 1, the natural periods of the initial shape structure are all $<0.5 \mathrm{~s}$. Hence, it is expected that sine waves with the periods shorter than $0.5 \mathrm{~s}$ are relatively critical as input waves. The sine waves are input for duration often times longer than each sine wave period to enable the system response to attain steady state.

\subsection{Design variables}

This paper considers two different optimization problems. One is the case of a system without any TMDs; this is a single objective optimization of the structural shape. The other is the case of a system with two TMDs; this is the simultaneous optimization of the structural shape and control system. In both cases, the control point coordinates of a Bézier surface are selected as design variables. In addition, the mass, damping, and stiffness of the TMDs are included in the design variables in the second case, which is a system with TMDs.

\subsection{Constraints}

One of the aims of this paper is to satisfy the intended design of an architectural designer with respect to a structural shape. This is realized through the optimization process by setting constraints for the variation between the positions of each node of the initial and optimal shapes. In this paper, the maximum variation is set to one-fourth of the structure span.

\subsection{Optimization problem}

The optimization problem setting is shown in Table 3.

Here $u_{i, j}(t), u_{g j}(t), x$, and $x_{0}$ are the displacement of the th node under $j$ th sine wave input, ground displacement of $j$ th sine wave input, and nodal position vectors of the optimal and initial designs, respectively. In the previous and proposed methods, the maximum number of $j$ is two and five, respectively. $\boldsymbol{B}_{0,0}, \boldsymbol{B}_{0,1}, \ldots, \boldsymbol{B}_{4,4}$ are the control point vectors of a Bézier surface of order $(4,4) . \boldsymbol{m}_{\mathrm{TMD}}, \boldsymbol{c}_{\mathrm{TMD}}$, and $\boldsymbol{k}_{\mathrm{TMD}}$ are the mass, damping, and stiffness vector of TMDs, respectively.

\section{OPTIMIZATION RESULTS}

\subsection{Results of sine wave input analyses}

Figure 3 shows the examples of the results of sine wave input analyses. The optimization aims to minimize the maximum absolute response value to sine wave inputs in the steady state, such as shown in Fig. 3. 
Table 3: Optimization problem setting.

\begin{tabular}{lcc}
\hline & System without TMDs & System with TMDs \\
\hline Objective function & $J=\max _{t, i, j}\left\{\ddot{u}_{i, j}(t)+\ddot{u}_{\mathrm{g} j}(t)\right\}$ & $J=\max _{t, i, j}\left\{\ddot{u}_{i, j}(t)+\ddot{u}_{\mathrm{g} j}(t)\right\}$ \\
Constraints & $\left\|x-x_{0}\right\|_{<\min \left(\frac{X}{4}, \frac{Y}{4}, \frac{Z}{4}\right)}$ & $\left\|x-x_{0}\right\|_{<\min }\left(\frac{X}{4}, \frac{Y}{4}, \frac{Z}{4}\right)$ \\
Design variables & $\boldsymbol{B}_{0,0}, \boldsymbol{B}_{0,1,}, \ldots, \boldsymbol{B}_{4,4}$ & $\boldsymbol{B}_{0,0}, \boldsymbol{B}_{0,1}, \ldots, \boldsymbol{B}_{4,4}, \boldsymbol{m}_{\mathrm{TMD}}, \boldsymbol{c}_{\mathrm{TMD}}, \boldsymbol{k}_{\mathrm{TMD}}$ \\
\hline
\end{tabular}

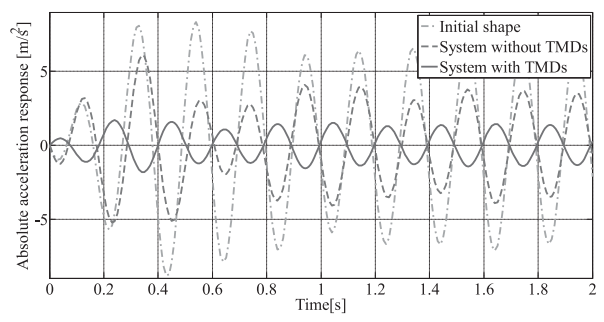

(a) Period: $0.2 \mathrm{~s}$

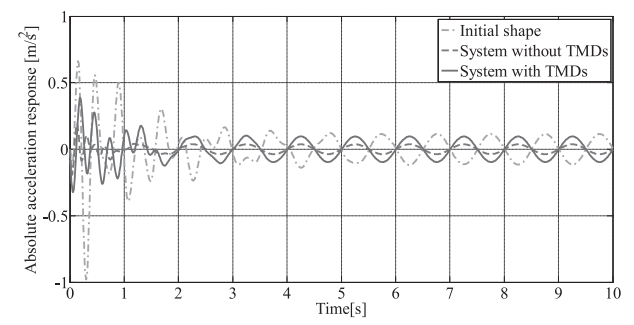

(b) Period: $1.0 \mathrm{~s}$

Figure 3: Examples of the results of sine wave input analyses. Period: (a) $0.2 \mathrm{~s}$ and (b) $1.0 \mathrm{~s}$.

Table 4: Natural periods (first to fifth) of the optimal design in the case of a system without TMDs.

\begin{tabular}{lccccc}
\hline & \multicolumn{5}{c}{ Natural period (s) } \\
\cline { 2 - 6 } Period of sine wave (s) & 1st & 2nd & 3rd & 4th & 5th \\
\hline 0.1 & 0.3789 & 0.2607 & 0.2168 & 0.1806 & 0.1308 \\
0.2 & 0.3270 & 0.2523 & 0.1585 & 0.1534 & 0.1303 \\
0.3 & 0.3706 & 0.2473 & 0.2201 & 0.1709 & 0.1416 \\
0.5 & 0.3598 & 0.2526 & 0.2099 & 0.1708 & 0.1269 \\
1.0 & 0.2781 & 0.2278 & 0.1707 & 0.1507 & 0.1356 \\
2.0 & 0.3355 & 0.2300 & 0.1866 & 0.1658 & 0.1391 \\
5.0 & 0.3493 & 0.2380 & 0.2128 & 0.1593 & 0.1247 \\
Previous method & 0.3424 & 0.2875 & 0.2132 & 0.1680 & 0.1458 \\
Proposed method & 0.4505 & 0.3165 & 0.2293 & 0.1780 & 0.1674 \\
\hline
\end{tabular}

The natural periods of lower modes of the system without and with TMDs for seven sine wave inputs are shown in Tables 4 and 5. In addition, the natural periods of the proposed method are also shown in Tables 4 and 5 .

The optimal parameters of two TMDs derived by GAs are shown in Table 6 . These can be derived directly through a GA optimization method. 
Table 5: Natural periods (first to fifth) of the optimal design in the case of a system with TMDs.

\begin{tabular}{lccccc}
\hline & \multicolumn{5}{c}{ Natural period (s) } \\
\cline { 2 - 6 } Period of sine wave (s) & 1st & 2nd & 3rd & 4th & 5th \\
\hline 0.1 & 0.3751 & 0.2626 & 0.2268 & 0.1813 & 0.1385 \\
0.2 & 0.3620 & 0.2567 & 0.1745 & 0.1620 & 0.1309 \\
0.3 & 0.3832 & 0.2444 & 0.2119 & 0.1738 & 0.1301 \\
0.5 & 0.3991 & 0.2688 & 0.2147 & 0.1691 & 0.1652 \\
1.0 & 0.3144 & 0.2725 & 0.2358 & 0.1584 & 0.1442 \\
2.0 & 0.4164 & 0.3107 & 0.2159 & 0.1829 & 0.1736 \\
5.0 & 0.3763 & 0.2604 & 0.2078 & 0.1769 & 0.1346 \\
Previous method & 0.3567 & 0.2774 & 0.2216 & 0.1772 & 0.1394 \\
Proposed method & 0.2165 & 0.1959 & 0.1948 & 0.1839 & 0.1552 \\
\hline
\end{tabular}

Table 6: Optimal parameters of two TMDs.

\begin{tabular}{lcc} 
& TMD 1 & TMD 2 \\
\hline Controlled mode & 1st & 3rd \\
Mass (kg) & 10.03 & 8.910 \\
Damping (Ns/m) & 3.100 & 4.450 \\
Stiffness (N/m) & 63.73 & 148.2 \\
\hline
\end{tabular}

Using the proposed method, the fundamental period becomes longer than that for the initial shape $(0.3948 \mathrm{~s})$ for a system without TMDs $(0.4505 \mathrm{~s})$ and becomes shorter than that for the initial shape for a system with TMDs $(0.2165 \mathrm{~s})$, as shown in Tables 4 and 5. For a system with TMDs, two TMDs are applied to reduce the maximum response of the 1st and 3rd modes, as shown in Table 6 . According to the objective function shown in Table 3, the vibration modes to be reduced include not only the 1 st and 3rd modes but also the 2nd, 4th, and 5 th modes. Therefore, the optimized design does not result in a shorter natural period; the 4 th and 5th natural periods become longer than the 4th and 5th natural periods of the initial shape. Instead, it produces a narrower range of the five natural periods, which improves the effectiveness of the two TMDs in damping the responses to the sine wave inputs with the same period as the 1st through 5th natural periods. For a system without TMDs, the optimal design produces a more extensive range of natural periods to prevent an increase in the response due to simultaneous excitations of multiple modes for a sine wave input.

\subsection{Response under seismic ground motion}

To confirm the seismic performance of the structural shape and effectiveness of the TMD control system, time history analyses were conducted. Four records, El Centro 1940, Taft 1952, Hachinohe 1968, and Takatori 1995, were selected as input waves. The input waves were scaled so that the maximum velocity of each component becomes $0.25 \mathrm{~m} / \mathrm{s}$. The NS, 

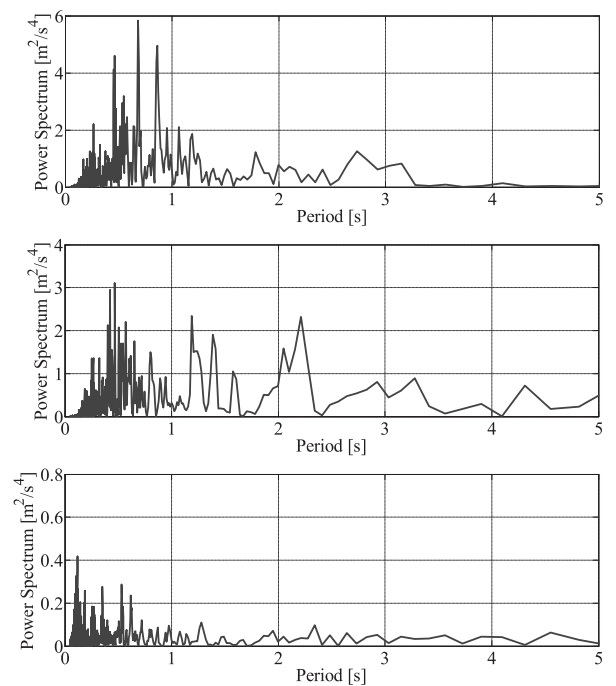

(a) El Centro
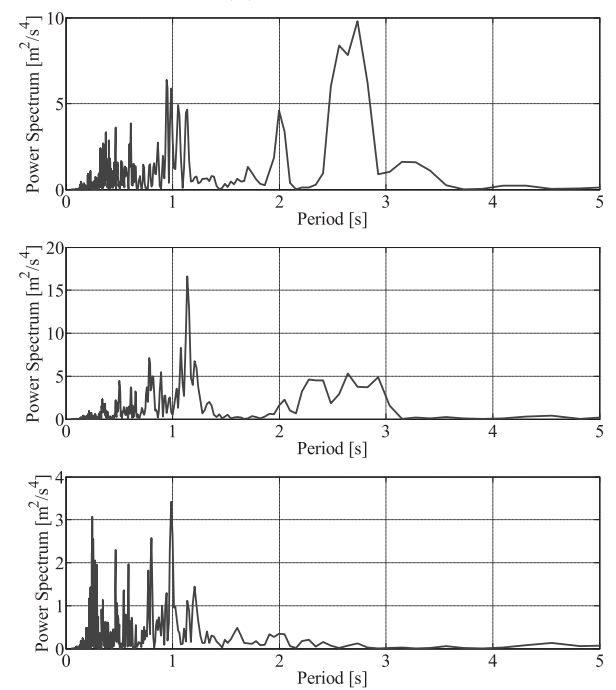

(c) Hachinohe
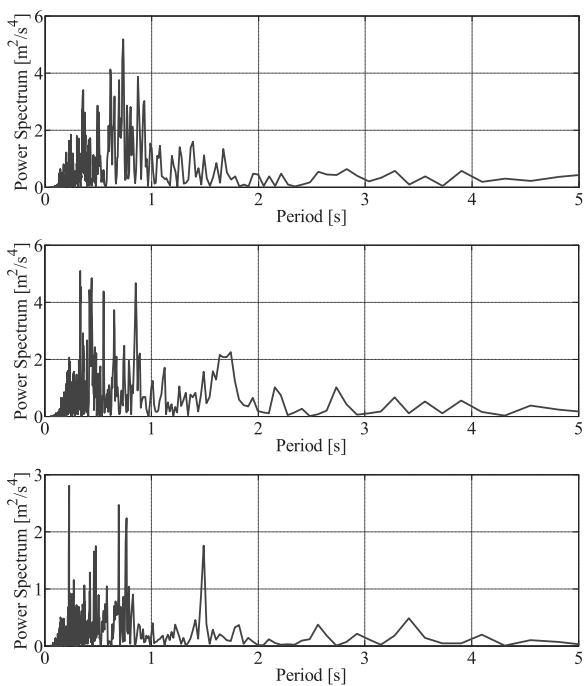

(b) Taft
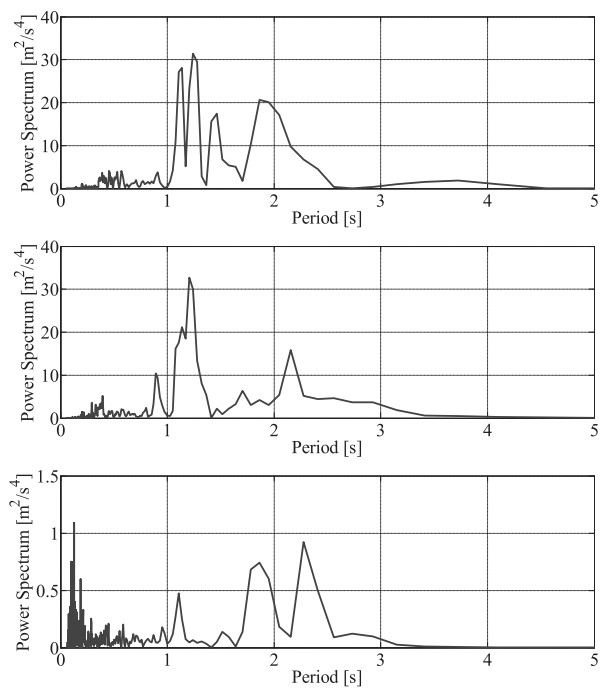

(d) Takatori

Figure 4: Power spectra of scaled input seismic waves (the top, middle, and bottom show NS, EW, and UD components, respectively). (a) El Centro, (b) Taft, (c) Hachinohe, and (d) Takatori.

EW, and UD component waves are inputs in the X-, Y-, and Z-directions, respectively. The power spectra of four input waves are shown in Fig. 4. El Centro and Taft have shorter period components; on the other hand, Hachinohe and Takatori have longer period components.

The maximum response values in the time-history response analyses are shown in Tables 7-10. In Tables 7 and 8, the maximum response value when $0.2 \mathrm{~s}$ period sine wave is input is the smallest except the value of the proposed method in the case of a system with TMDs. On the other hand, it is not the best result in Tables 9 and 10. According to Fig. 4, El Centro and Taft waves have shorter period components; therefore, optimization results using shorter 
Table 7: Maximum response values under El Centro wave.

\begin{tabular}{|c|c|c|c|}
\hline \multirow{2}{*}{$\begin{array}{l}\text { Period of sine wave } \\
\text { in optimization (s) }\end{array}$} & \multicolumn{3}{|c|}{ Absolute acceleration of node $\left(\mathrm{m} / \mathrm{s}^{2}\right)$} \\
\hline & Initial shape & System without TMDs & System with TMDs \\
\hline 0.1 & \multirow{9}{*}{8.974} & 4.750 & 4.338 \\
\hline 0.2 & & 5.908 & 3.640 \\
\hline 0.3 & & 8.171 & 8.483 \\
\hline 0.5 & & 7.177 & 5.818 \\
\hline 1.0 & & 4.892 & 4.204 \\
\hline 2.0 & & 3.918 & 5.236 \\
\hline 5.0 & & 8.056 & 6.433 \\
\hline Previous method & & 5.548 & 6.277 \\
\hline Proposed method & & 4.591 & 3.618 \\
\hline
\end{tabular}

Table 8: Maximum response values under Taft wave.

\begin{tabular}{|c|c|c|c|}
\hline \multirow{2}{*}{$\begin{array}{l}\text { Period of sine wave } \\
\text { in optimization (s) }\end{array}$} & \multicolumn{3}{|c|}{ Absolute acceleration of node $\left(\mathrm{m} / \mathrm{s}^{2}\right)$} \\
\hline & Initial shape & System without TMDs & System with TMDs \\
\hline 0.1 & \multirow{9}{*}{11.93} & 12.16 & 6.470 \\
\hline 0.2 & & 9.908 & 5.610 \\
\hline 0.3 & & 10.32 & 11.56 \\
\hline 0.5 & & 8.938 & 7.837 \\
\hline 1.0 & & 8.709 & 7.916 \\
\hline 2.0 & & 8.981 & 7.419 \\
\hline 5.0 & & 10.73 & 9.081 \\
\hline Previous method & & 7.617 & 9.134 \\
\hline Proposed method & & 8.543 & 4.423 \\
\hline
\end{tabular}

Table 9: Maximum response values under Hachinohe wave.

\begin{tabular}{|c|c|c|c|}
\hline \multirow{2}{*}{$\begin{array}{l}\text { Period of sine wave } \\
\text { in optimization (s) }\end{array}$} & \multicolumn{3}{|c|}{ Absolute acceleration of node $\left(\mathrm{m} / \mathrm{s}^{2}\right)$} \\
\hline & Initial shape & System without TMDs & System with TMDs \\
\hline 0.1 & \multirow{9}{*}{11.21} & 5.344 & 8.103 \\
\hline 0.2 & & 9.470 & 7.634 \\
\hline 0.3 & & 10.56 & 12.61 \\
\hline 0.5 & & 10.78 & 8.506 \\
\hline 1.0 & & 6.579 & 6.654 \\
\hline 2.0 & & 6.734 & 7.532 \\
\hline 5.0 & & 10.59 & 10.46 \\
\hline Previous method & & 8.486 & 9.045 \\
\hline Proposed method & & 6.353 & 3.364 \\
\hline
\end{tabular}


Table 10: Maximum response values under Takatori wave.

\begin{tabular}{|c|c|c|c|}
\hline \multirow{2}{*}{$\begin{array}{l}\text { Period of sine wave } \\
\text { in optimization (s) }\end{array}$} & \multicolumn{3}{|c|}{ Absolute acceleration of node $\left(\mathrm{m} / \mathrm{s}^{2}\right)$} \\
\hline & Initial shape & System without TMDs & System with TMDs \\
\hline 0.1 & \multirow{9}{*}{18.07} & 8.076 & 11.23 \\
\hline 0.2 & & 16.10 & 10.04 \\
\hline 0.3 & & 14.57 & 18.17 \\
\hline 0.5 & & 13.90 & 13.33 \\
\hline 1.0 & & 9.555 & 11.19 \\
\hline 2.0 & & 11.31 & 11.37 \\
\hline 5.0 & & 12.58 & 15.88 \\
\hline Previous method & & 11.51 & 12.99 \\
\hline Proposed method & & 8.893 & 3.883 \\
\hline
\end{tabular}
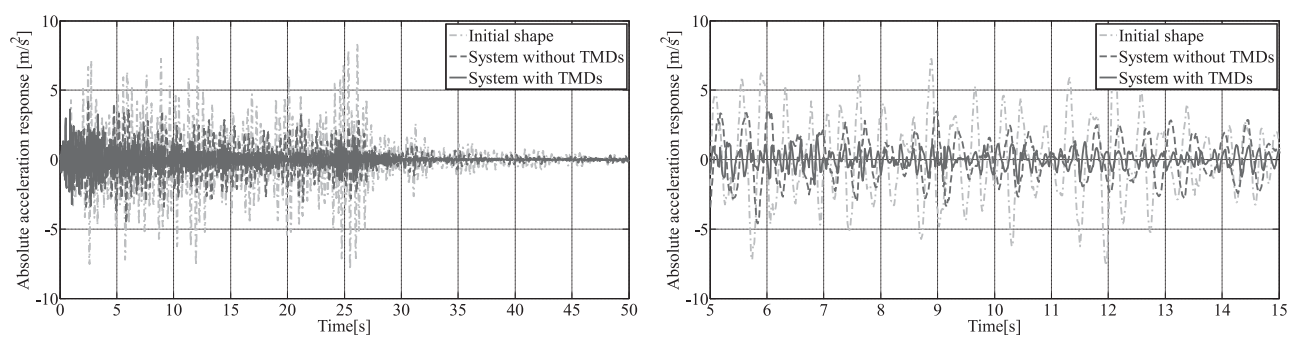

Figure 5: Results of time-history response analyses under El Centro wave (the right: the time span including the peak response value is magnified).

period sine wave inputs show better performance than others under these two input waves. However, under Hachinohe and Takatori waves, the optimization results do not show the best performance because these two input waves have longer period components. Thus, it can be concluded that the natural periods of the structure are appropriate for the period of the input sine wave in optimization as adopted in the proposed method. However, the results of the previous method are worse than the results of single sine wave input in many cases. On the other hand, all of the maximum response values of the proposed method are smaller than any of other results. Thus, it can be concluded that the optimal design of the proposed method show satisfactory performance under input ground motions with various frequency characteristics.

The results of the time-history response analyses are shown in Figs 5-8. In the right figures of Figs 5-8, the time span is magnified in which the peak response value is included. The proposed optimization method is confirmed to be effective for reducing the seismic response. In particular, the peak response values of both optimal shapes without and with TMDs are significantly reduced compared with that of the initial shape. However, in some cases, the response of the optimal shape with TMDs is larger than that without TMDs. This is possible because the frequency characteristic of the input ground motion affects the response; further research should be conducted using various input ground motions. In addition, the maximum response value of the optimal shape with TMDs is smaller than that without TMDs. Thus, the 

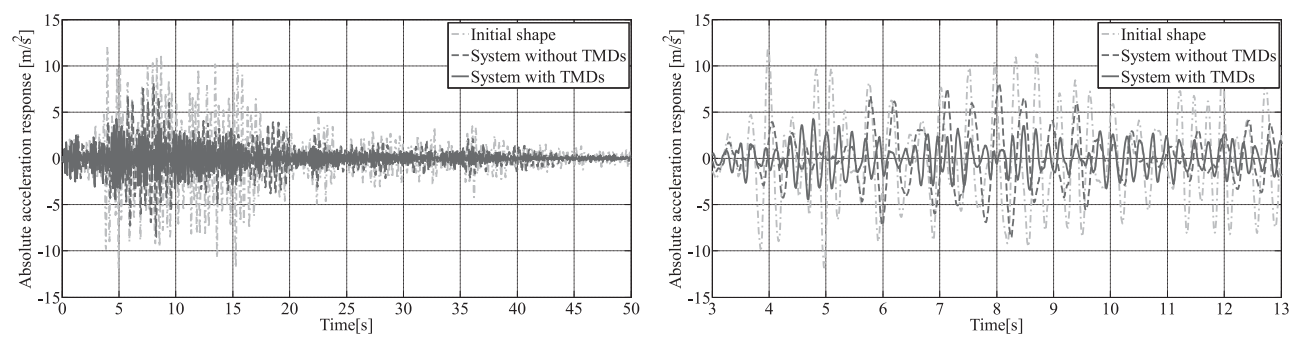

Figure 6: Results of time-history response analyses under Taft wave (the right: the time span including the peak response value is magnified).
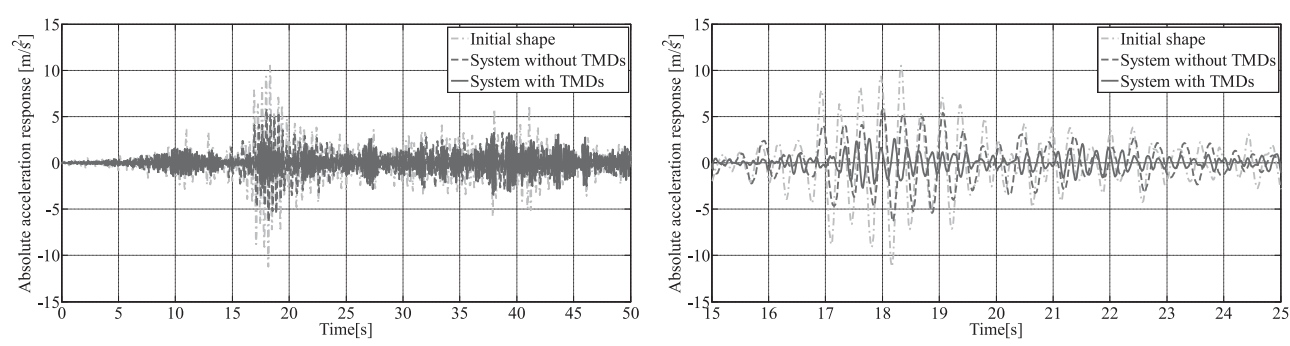

Figure 7: Results of time-history response analyses under Hachinohe wave (the right: the time span including the peak response value is magnified).
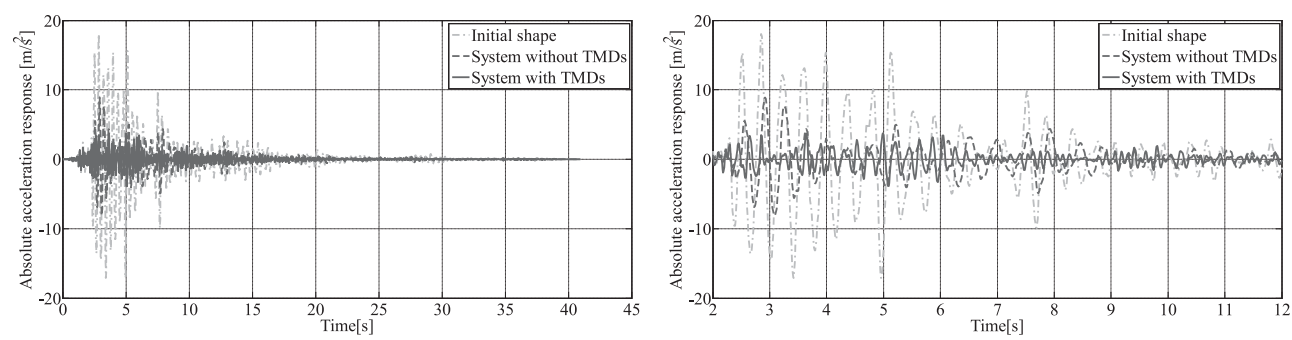

Figure 8: Results of time-history response analyses under Takatori wave (the right: the time span including the peak response value is magnified).

effectiveness of TMDs is also confirmed, and the proposed optimization method can be concluded to be useful for the design of an asymmetric large-scale space frame.

\subsection{Results of the optimization}

Figure 9 shows the initial shape, and the asterisk (*) shows the node that has the maximum response in the time-history response analysis under conditions of the seismic ground motion explained in the next section. In this case, the maximum response occurred in the up-down (the Z-direction) component. The optimal shapes in the case of a system without and with TMDs are shown in Figs 10 and 11, respectively. In these cases, the maximum response also occurred in the up-down direction (the Z-direction) component. 


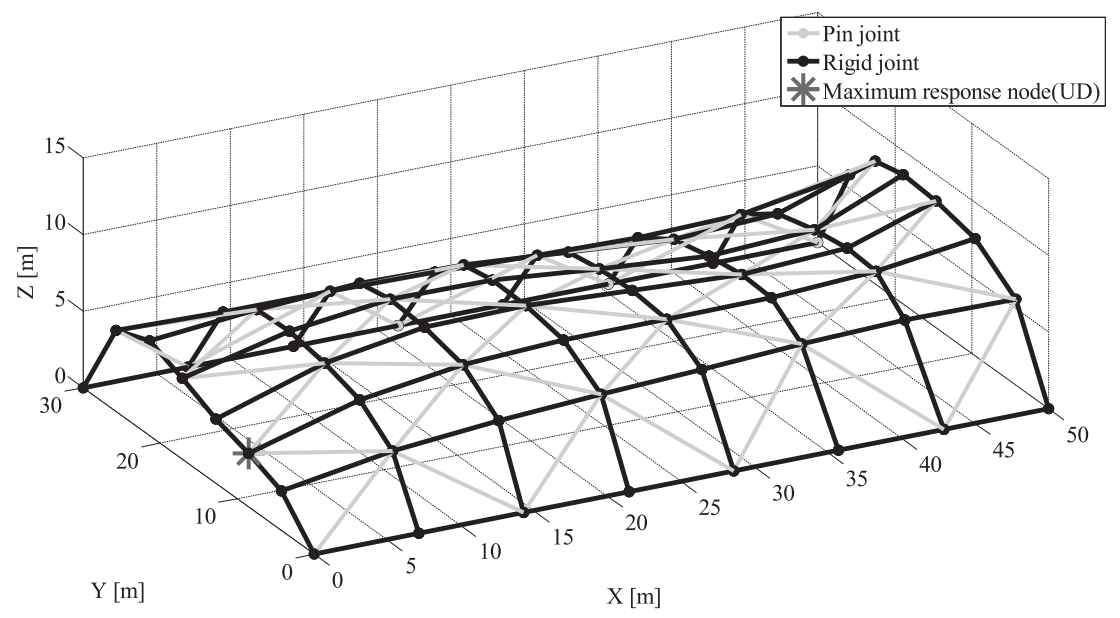

Figure 9: Initial shape and the maximum response node.

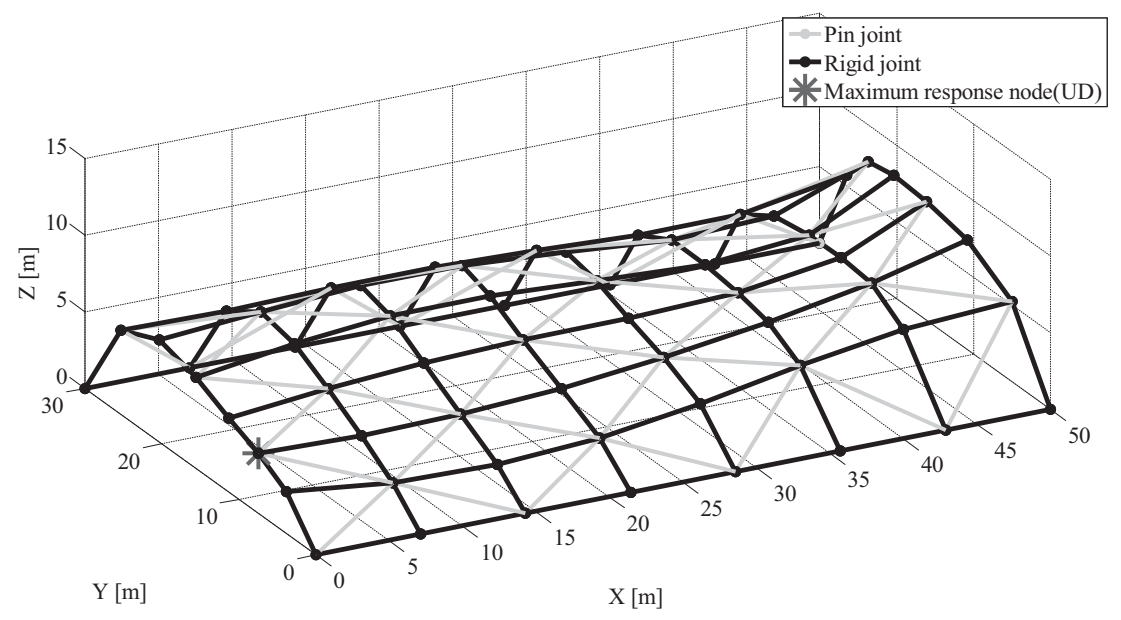

Figure 10: Optimal shape in the case of a system without TMDs.

TMDs enable us to keep a structural shape that implements the intended design of an architectural designer when used as a seismic control system.

\section{CONCLUSIONS}

This study proposed a method to obtain optimal design variables related to both structural shape and control devices simultaneously for asymmetric large-scale space frames with TMDs. As design variables, control node coordinates of a Bézier surface, which describes nodal coordinates of joints of the space frame, and parameters of TMDs were considered. The total number of design variables could be reduced by using a Bézier surface, and the change of the nodal coordinates of the space frame was constrained to keep a similar shape to the initial design provided by an architect. With respect to the objective function of the 


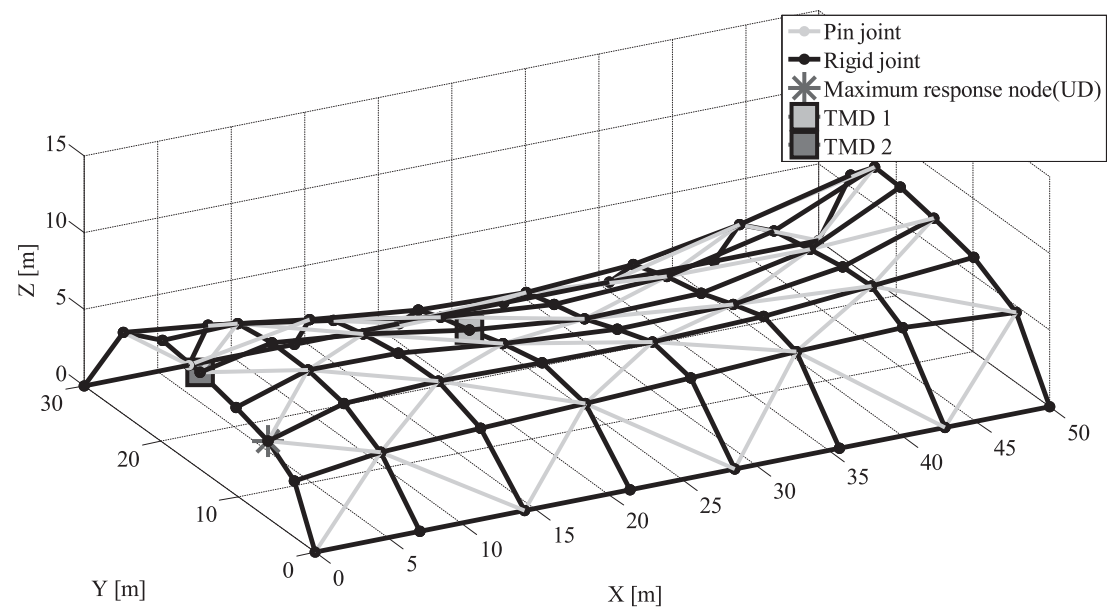

Figure 11: Optimal shape in the case of a system with TMDs.

optimal design problem, the maximum absolute acceleration response to five sine wave inputs was adopted, of which periods were the same as the natural periods of the large modal participation factors. The method employed GA in the optimization.

In a validity study, a space frame structure with 64 joint nodes and 161 beams was examined. It is confirmed that the proposed method could provide the optimal shape that comparatively reduces the maximum absolute acceleration response to sine wave inputs with the periods of 0.1 , $0.2,0.3,0.5,1.0,2.0$, and $5.0 \mathrm{~s}$. In addition, a time-history analysis was conducted using four scaled input ground motions: El Centro 1940, Taft 1952, Hachinohe 1968, and Takatori 1995. The results also confirmed that the maximum absolute acceleration responses to the seismic ground motions were significantly reduced in the optimal shapes; the employment of TMDs enabled the reduction of the peak response value and maintained similarity to the initial shape.

Although this study focused on TMDs as vibration control devices, other control systems, such as semi-active and active control systems, should be considered in future research. In introducing a semi-active or active control system to the large-scale space frame, the optimization of a whole structure including both the roof and substructure should be considered. In addition, a comparison of these control systems based on their life-cycle cost is expected. Furthermore, it is important to specify clear constraints that can help architectural designers to design more esthetic and high-performance structures.

\section{ACKNOWLEDGMENT}

This work is supported in part by a Grant-in-Aid for the Leading Graduate School Program for 'Science for Development of Super Mature Society' from the Ministry of Education, Culture Sports, Science and Technology in Japan.

\section{REFERENCES}

[1] Architecture Institute of Japan, Report on the Hanshin-Awaji Earthquake Disaster, Building Series, Volume 3: Structural Damage to Steel Buildings, Structural Damage to Shell and Spatial Structures, Structural Damage to Storage Tanks and Their Supports, 1998 (in Japanese).

[2] Architecture Institute of Japan, Preliminary Reconnaissance Report of the 2011 Tohoku-Chiho Taiheiyo-Oki Earthquake. Springer, 2012. 
[3] Kaynia, A.M., Veneziano, D. \& Biggs, J.M., Seismic effectiveness of tuned mass dampers. Journal of the Structural Division, 107(8), pp. 1465-1484, 1981.

[4] Lin, C.-C., Hu, R.-Y. \& Wang, J.-J., Optimal design of passive tuned mass dampers for seismic structures. 33rd Structures, Structural Dynamics and Materials Conference, Vol. 33, pp. 1497-1503, 1992.

[5] Johnson, J.G., Reavelley, L.D. \& Pantelides, C., A rooftop tuned mass damper frame. Earthquake Engineering \& Structural Dynamics, 32(6), pp. 965-984, 2003. doi: http:// dx.doi.org/10.1002/eqe. 257

[6] Kusunoki, T., Xue, S. \& Yamada, M., Study of seismic response and vibration control of single-layer latticed domes using TMD. Journal of Structural Engineering, 41B, pp. 17-22, 1995 (in Japanese).

[7] Yoshinaka, S. \& Kawaguchi, K., Vibration control of spatial structures using spatially distributed MTMDs. Memoirs of the Faculty of Engineering, Osaka City University, 49, pp. 19-28, 2008.

[8] Ramm, E., Bletzinger, K.U. \& Reitinger, R., Shape optimization of shell structures. Revue Européenne des Éléments, 2(3), pp. 377-398, 1993.

[9] Ohsaki, M., Nakamura, T. \& Kohiyama, M., Shape optimization of a double-layer space truss described by a parametric surface. International Journal of Space Structures, 12(2), pp. 109-119, 1997.

[10] Goldberg, D.E., Genetic Algorithm in Search Optimization and Machine Learning. New York: Addison-Wesley, 1989.

[11] Jenkins, W.M., Towards structural optimization via the genetic algorithm. Computers \& Structures, 40(5), pp. 1321-1327, 1991. doi: http://dx.doi.org/10.1016/00457949(91)90402-8

[12] Rajeev, S. \& Krishnamoorthy, C., Discrete optimization of structures using genetic algorithms. Journal of Structural Engineering, 118(5), pp. 1233-1250, 1992. doi: http:// dx.doi.org/10.1061/(ASCE)0733-9445(1992)118:5(1233)

[13] Rajan, S.D., Sizing, shape, and topology design optimization of trusses using genetic algorithm. Journal of Structural Engineering, 121(10), pp. 1480-1487, 1995. doi: http://dx.doi.org/10.1061/(ASCE)0733-9445(1995)121:10(1480)

[14] Ohsaki, M., Genetic algorithm for topology optimization of trusses. Computers \& Structures, 57(2), pp. 219-225, 1995. doi: http://dx.doi.org/10.1016/0045-7949(94)00617-C

[15] Camp, C., Pezeshk, S. \& Cao, G., Optimized design of two-dimensional structures using a genetic algorithm. Journal of Structural Engineering, 124(5), pp. 551-559, 1998. doi: http://dx.doi.org/10.1061/(ASCE)0733-9445(1998)124:5(551)

[16] Erbatur, F., Hasançebi, O., Tütüncü, I. \& Kılıç, H., Optimal design of planar and space structures with genetic algorithms. Computers \& Structures, 75(2), pp. 209-224, 2000. doi: http://dx.doi.org/10.1061/(ASCE)0733-9445(1998)124:5(551)

[17] Dan, M. \& Kohiyama, M., Simultaneous optimization of structural shape and control system of large-scale space frame. WIT Transactions on the Built Environment, 132, 2013, ISSN 1743-3509, doi.10.2495/ERES130141.

[18] Den Hartog, J.P., Mechanical Vibration, 4th edn, McGraw-Hill: New York, 1956.

[19] Ikago, K., Saito, K. \& Inoue, N., Seismic control of single-degree-of-freedom structure using tuned viscous mass damper. Earthquake Engineering and Structural Dynamics, 41, pp. 453-474, 2011. doi: http://dx.doi.org/10.1002/eqe.1138

[20] Kato, S., Nakazawa, S., Uchikoshi, M. \& Mukaiyama, Y., Response reducing effect of seismic isolation system installed between large dome and lower structure. Proceedings of 6th Asian Pacific Conference on Shell and Spatial Structures, Seoul, Korea, pp. 323-330, 2001. 